\title{
Application of Time Recursive Processing for the Development of a Time/Phase Shifter
}

\author{
Djurdje M. Perisic \\ Faculty of Information Technology \\ Slobomir P University, SPU \\ Slobomir, Bjeljina, Republic of Srpska, BIH \\ djurdje@beotel.rs
}

\author{
Miroslav Bojovic \\ Faculty of Electrical Engineering \\ University of Belgrade \\ Belgrade, Serbia \\ mbojovic@etf.bg.ac.rs
}

\begin{abstract}
This paper describes a powerful digital time and/or phase shifter of pulse rates, based on Time Recursive Processing. It can function either as a time shifter or as a phase shifter. The circuit can generate precise shifting for a pulse rate with a constant input period, but it is also capable to shift a pulse rate whose period is a ramp function. Besides that, the shifter can be used in tracking and prediction applications. The shifter is described by recursive equations as a linear discrete system. All mathematical analyses are made using the $Z$ transform. Computer simulations are employed to prove the correctness of the mathematical analysis. The realization of the shifter is described and, to demonstrate the shifter functioning, actual oscilloscope screenshots are presented.
\end{abstract}

Keywords- time shifter; phase shifter; digital circuit; PLL

\section{INTRODUCTION}

The field of Time Recursive Processing (TRP) has been described in detail in previous works such as [1-9]. The TRP approach is based on the measurement and processing of the input and output periods and time differences between them. Although the first idea was simply to discover a new kind of Phase Locked Loop (PLL) and Frequency Locked Loop (FLL), it turned out that TRP is suitable for a much broader range of applications. Namely, most of the circuits described in [1-9], possess some of the properties of either classic Phase Locked Loop (PLL) or classic Frequency Locked Loop (FLL). However, due to their properties, Time Recursive PLL (TR PLL) and Time Recursive FLL (TR FLL) exceed the applicability and functionality of classic PLL and FLL, improving the efficiency and expanding the scope and diversity of their applications. Different applications include phase shifting in [1, 2], the development of digital frequency synthesizers [3], noise rejection applications [4-6] and a wide range of tracking and prediction [4, 5, 7]. All the algorithms described are very suitable for usage in a software form, like for example a software predictor [8]. Although a very complex system, consisting of a lot of subsystems, it can also function as a FLL, whose realization is based on the same technique [9].

TR PLL and TR FLL represent a completely new approach both in theory and in implementation and application. For example, the measurement of phase, frequency or amplitude, which are used in classic PLL and FLL, are changed by the measurement of time, which is much more convenient and more precise. Let us remember that the classic PLL tends to equalize both frequency and phase between the input and the output signals, reducing the phase difference to zero. Unlike PLL, classic FLL tends to equalize the frequency, without taking care of the phase difference. When FLL reaches the stable state, the phase difference between the input and output signals depends on the initial variable conditions and, possibly, it depends on the system parameters. In other words, the phase difference is the random variable. According to $[1,2]$ some of TR PLLs possess the ability to regulate the phase difference by either system parameters or by output control. This is an important feature of the TR PLL, which can be utilized to generate phase differences of any value. Note that the phase difference of any PLL cannot depend on the initial conditions of the variables.

In this paper, a new model of a time/phase shifter, based on TRP, is described. It provides phase shifting in a band without limitations which is controlled by the clock frequency. Under some system conditions, it can also provide time shifting linearly dependent on the value of an outside control. In comparison with the previous phase shifters of this type, described in $[1,2]$, this phase shifter can generate the proper shifting even in case the input period is a ramp function.

\section{MATHEMATICAL DESCRIPTION OF THE SHIFTER}

One general case of the time relation between an input signal Sin and an output signal Sop of the shifter, is shown in Figure 1. The periods $T I_{0}, T I_{1}, \ldots T I_{\mathrm{k}}, T I_{\mathrm{k}+1}$, and $T O_{0}$, $T O_{1}, \ldots T O_{\mathrm{k}}, T O_{\mathrm{k}+1}$, as well as the time differences $\tau_{0}, \tau_{1}$, $\tau_{2} \ldots \ldots \tau_{k}, \tau_{k+1}$, occur at discrete times respectively $t_{0}, t_{1}, t_{2}, \ldots . . t_{k}$, $t_{k+1}$. The discrete times $t_{0}, t_{1}, t_{2}, \ldots t_{k}, t_{k+1}$ are defined by the falling edges of the pulses of Sop in Figure 1. Note that, unlike the classic PLL, all variables are distributed in time in Figure 1. The natural recursive relation (1), between the variables, yields from Figure 1. It was supposed that the time difference $\tau$ is positive if the output signal leads the input signal. Correspondingly, in case that the input signal leads, time difference $\tau$ will take a negative value through the analysis. The main algorithm of the circuit is presented in (2), where " $T_{k}$ ", as an outside control word, represents a step function and "a" and "m" are the system parameters. According to (1) and 
(2), the circuit has two output variables, which describe its behavior:

$$
\begin{gathered}
\tau_{k+1}=\tau_{k}+T O_{k}-T I_{k} \\
T O_{k+1}=\mathrm{a} \cdot T I_{k}+T_{k}+m \cdot \tau_{k+1}
\end{gathered}
$$

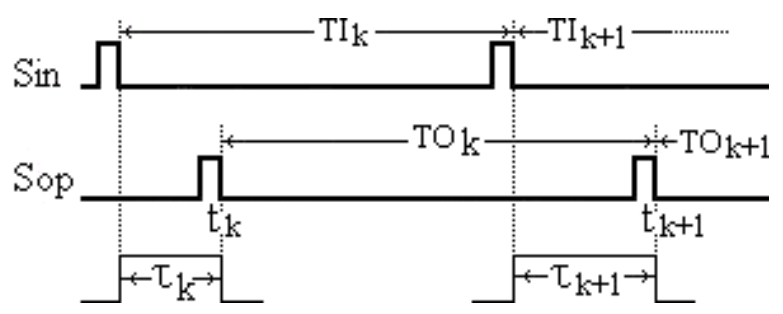

Fig. 1. Time relations between all variables of the circuit.

The output variables are $\tau(k+1)=\mathrm{f}[T I(k)]$ and $T O(k+1)=$ $\mathrm{f}[T I(k)]$. Note that, because of simplicity, $T O(k), T I(k)$ and $\tau(k)$ are denoted in the article as $T O_{k}, T I_{k}$ and $\tau_{k}$. It is now necessary to find the $\mathrm{Z}$ transform of the output variables $\tau(k+1)$ and $T O(k+1)$ in order to analyze the properties of the shifter described. The $Z$ transform of (1) and (2) are given by respectively (3) and (4). Time constants $T O_{0}$ and $\tau_{0}$ are the initial values of the output variables $T O(k)$ and $\tau(k)$, which appear at discrete time $t_{0}=0$.

$$
\begin{gathered}
z \cdot \tau(z)-z \cdot \tau_{0}=\tau(z)+T O(z)-T I(z) \\
z T O(z)-z T O_{0}=\mathrm{a} T I(z)+T(z)+\left[z \tau(z)-z \tau_{0}\right] \cdot m
\end{gathered}
$$

Calculating $\tau(z)$ from (3) and using it into (4), gives:

$T O(\mathrm{z})=T I(\mathrm{z}) \frac{\mathrm{z}(\mathrm{a}-\mathrm{m})-\mathrm{a}}{\mathrm{z}(\mathrm{z}-\mathrm{m}-1)}+\frac{T(\mathrm{z}) \cdot(\mathrm{z}-1)+\tau_{0} \mathrm{mz}+T O_{0} \mathrm{z}}{\mathrm{z}(\mathrm{z}-\mathrm{m}-1)}$

In the same way, changing $T O(z)$ from (5) to (3), gives:

$\tau(z)=-T I(z) \frac{z-\mathrm{a}}{z(\mathrm{z}-\mathrm{m}-1)}+\frac{T(\mathrm{z})+\tau_{0} \cdot z(\mathrm{z}-m)+T O_{0} \cdot z}{z(\mathrm{z}-\mathrm{m}-1)}$

Two transfer functions, presented by (7) and (8), describing the output variables of the shifter in dependence on the input period, can be defined from respectively (5) and (6).

$$
\begin{gathered}
H_{T O}(z)=\frac{T O(z)}{T I(z)}=\frac{\mathrm{z}(\mathrm{a}-\mathrm{m})-\mathrm{a}}{\mathrm{z}(\mathrm{z}-\mathrm{m}-1)} \\
H_{\tau}(z)=\frac{\tau(z)}{T I(z)}=-\frac{z-\mathrm{a}}{z(\mathrm{z}-\mathrm{m}-1)}
\end{gathered}
$$

\section{A. Step analyzes of the circuit}

Step analysis will discover under which conditions the circuit can possess the properties of a time/phase shifter. Providing that the control time function $T(k)=T=$ const., let us suppose that a step function, $T I(k)=T I=$ const., is applied to the input. If we change the $\mathrm{Z}$ transforms $T(z)=T \cdot z /(z-1)$ and $T I(z)$ $=T I \cdot z /(z-1)$ into (5) and (6), we will get the $\mathrm{Z}$ transform of the output variables for this input. Changing $T(z)$ and $T I(z)$ into (5) and using the final value theorem, it is possible to find the final value of the output period in the time domain as $T O_{\infty}=$ $\lim T O(k)$ if $k \rightarrow \infty$, using $T O(z)$, as shown in (9). Applying (9), $T O_{\infty}$ is calculated as shown in (10). To complete the information about the system properties, it is necessary to determine $\tau_{\infty}$, i.e. the final value of $\tau(k)$. If $T(z)$ and $T I(z)$ are changed into (6), using the final value theorem in the same way like for $T O_{\infty}, \tau_{\infty}$ can be determined using (11). Applying (11), $\tau_{\infty}$ is calculated and shown in (12).

$$
\begin{gathered}
T O_{\infty}=\lim [(z-1) \cdot T O(z)]_{z \rightarrow 1} \\
T O_{\infty}=\lim [T O(k)]_{k \rightarrow \infty}=T I \\
\tau_{\infty}=\lim \tau(k)_{k \rightarrow \infty}=\lim [(z-1) \cdot \tau(z)]_{z \rightarrow 1} \\
\tau_{\infty}=T I(1-a) / m-T / m
\end{gathered}
$$

The expressions (10) and (12) are valued only if the shifter is the stable system i.e. if $\left|z_{1}\right|<1$ and $\left|z_{2}\right|<1$, where $z_{1}$ and $z_{2}$ are the poles of the transfer function $\mathrm{H}_{\mathrm{TO}}(\mathrm{z})$ or $\mathrm{H} \tau(\mathrm{z})$, given by (7) and (8). Since $z_{1}=0$, and $z_{2}=1+m$, it yields that the shifter is the stable system if (13) is satisfied. According to (10) and (12), it follows that the described shifter possesses the properties of a PLL. This conclusion comes out from the facts that the output period $T O_{\infty}$, for the stable system, equalizes the input period and that the time difference $\tau_{\infty}$ does not depend on the initial conditions $\tau_{0}$ and $T O_{0}$.

$$
-2<m<0
$$

\section{B. The time and/or phase shifting}

Looking at (12), we can notice that total value of $\tau_{\infty}$ consists of two parts. The first one is $T I(1-\mathrm{a}) / \mathrm{m}$. The term (1a) $/ \mathrm{m}$ indicates the portion of a period $T I$, or the number of periods $T I$, for which the input pulse rate is phase shifted. According to its nature, the first part represents the phase shifting. The system stability does not depend on the parameter "a", so that the value of either positive or negative phase shifting is unlimited. The second part is equal to "-T/m". It represents the pure time shifting, which is controlled by " $T$ ". It can also be ether positive or negative. Since control word " $T$ " does not affect on the system stability, this kind of shifting can be also unlimited. So, two kinds of shifting can be generated simultaneously. But they can be also generated separately. If $\mathrm{a}=1$, the first part in (12) does not take part in the shifting. At the same time, if control time function $T=0$, the second part in (12) has no effect on the shifting.

To prove the correctness of the previous analysis, simulations of the shifter functioning are presented. At the same time, the simulations are employed to enable a further insight into the procedure and into the physical meaning of the variables described. The simulations are also used to discover additional properties and possible efficient applications of the shifter. All discrete values in simulations were merged to form continuous curves. Note that all variables in the following diagrams were presented in time units. The time unit can be, $\mu \mathrm{sec}, \mathrm{msec}$ or any other, but assuming the same time units for $T I, T O, \tau$ and $T$. It was more suitable to use just "time unit" or abbreviated "t.u." in the text. It was more convenient to omit the indication "t.u." in diagrams. All simulations were made by Matlab, using (1) and (2). 
Let us first consider the time shifting, taking $a=1$. The locking procedure of the shifter is considered for three cases in Figure 2. The input is a step function $T I=10$ t.u. The parameter " $\mathrm{m} "$ and control word " $T$ " are chosen carefully in order to demonstrate the different properties of the shifter. All values of " $\mathrm{m}$ " and " $T$ ", as well as the values of the initial conditions $T O_{0}$ and $\tau_{0}$ are presented in Figure 2. It can be seen in Figure 2a, that for any value of "m" satisfying (13), the output period tends to the input period, but the transition time depends on the value of " $m$ ". For case Nr. 2, $(m=-1)$ the shifter is extremely fast. It reaches the stable state for only two steps. For case Nr. $1,(\mathrm{~m}=-0.85)$, and case $\mathrm{Nr} .3(\mathrm{~m}=-1.2)$, the shifter takes longer time to reach the stable state. The conclusion is that the greater deviation of parameter " $\mathrm{m}$ ", with respect to $\mathrm{m}=-1$, provides longer transition time of the shifter. Note that for all three cases, the calculation of $\tau_{\infty}=-T / \mathrm{m}$ agrees with $\tau_{\infty}$ which can be seen on the simulated curves. For instance for case Nr. 2, $\tau_{\infty}=-$ $\mathrm{T} / \mathrm{m}=-(-3) /-1=-3$. The same result can be seen in Figure $2 \mathrm{a}$. This agreement confirms the correctness of the simulation results and the correctness of the whole approach.

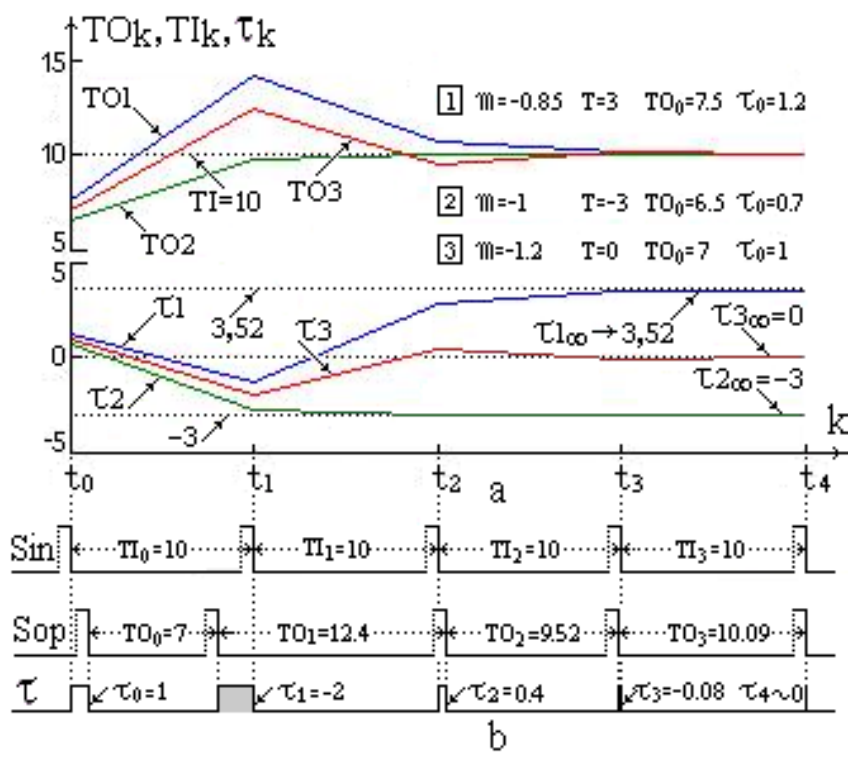

Fig. 2. Time shifting ( $\mathrm{a}=1$ for all of three cases): $a$. The output period tends to the input period and the final value of time difference $\tau_{\infty}=-T / \mathrm{m}, \mathrm{b}$. The case Nr. 3, in Figure 2a, is presented in the real time form. All periods of Sin, Sop and time differences $\tau_{\mathrm{k}}$ are calculated, showing the real time relation between them. For this case, $T=0\left(\tau_{\infty}=0\right)$ and the shifter functions as a classic PLL.

The merged discrete values for case Nr. 3, representing the output variables $\mathrm{TO}_{3}$ and $\tau_{3}$ in Figure $2 \mathrm{a}$, are shown in real time, in Figure $2 \mathrm{~b}$. This presentation, in forms of pulse rates, exhibits the real time relations between all input and output variables. It also helps to better understand the physical mining of the simulated curves. The calculated values of all variables are shown for every step. All values are taken from the simulation results, but they can be calculated manually, step by step, using (1) and (2). According to the mathematical analyzes and simulation results obtained, the shifter functions as classic
PLL for $T=0$ and $\mathrm{a}=1$. The negative values of the time differences $\tau_{\mathrm{k}}$ are shaded in Figure $2 \mathrm{~b}$.

Let us now consider the properties of the circuit in case that it functions as the phase shifter. In this case, according to (12), $T=0$ and $\tau_{\infty}=T I(1-\mathrm{a}) / \mathrm{m}$. The locking procedure of the shifter is simulated for three cases in Figure 3. The input is the step function $T I=10$ t.u. All values of "m" and "a", as well as the values of the initial conditions $T O_{0}$ and $\tau_{0}$ are presented in Figure 3. It can be seen in Figure 3a, that the role of parameter " $\mathrm{m}$ " is not changed. For any value of parameter " $\mathrm{m}$ " satisfying (13), the output period tends to the input period, but the transition time depends on the value of the parameter " $m$ ". For case $\mathrm{Nr} .2,(\mathrm{~m}=-1)$ the shifter reaches the stable state for only two steps. The conclusion is the same as in the case of the time shifter. The greater deviation of parameter "m", with respect to $\mathrm{m}=-1$, provides longer transition time of the shifter. Although the parameter " $\mathrm{m}$ " affects $\tau_{\infty}$, it is much more convenient to use "a" for the phase shifting. For case Nr. 2, a=1 and $\tau_{2 \infty}=0$, regardless of the values of "m". In this case phase shifter functions as a classic PLL. For case Nr. 1, a=1.16 (a>1) and $\tau_{1 \infty}=T I(1-\mathrm{a}) / \mathrm{m}=2$ t.u. The same result for $\tau_{1 \infty}$ can be seen in Figure $3 \mathrm{~b}$. For case Nr. 3, $\mathrm{a}=0.75(\mathrm{a}<1)$ and $\tau_{3 \infty}=T I(1-\mathrm{a}) / \mathrm{m}=-2$ t.u. The same result can be also seen in Figure $3 b$.

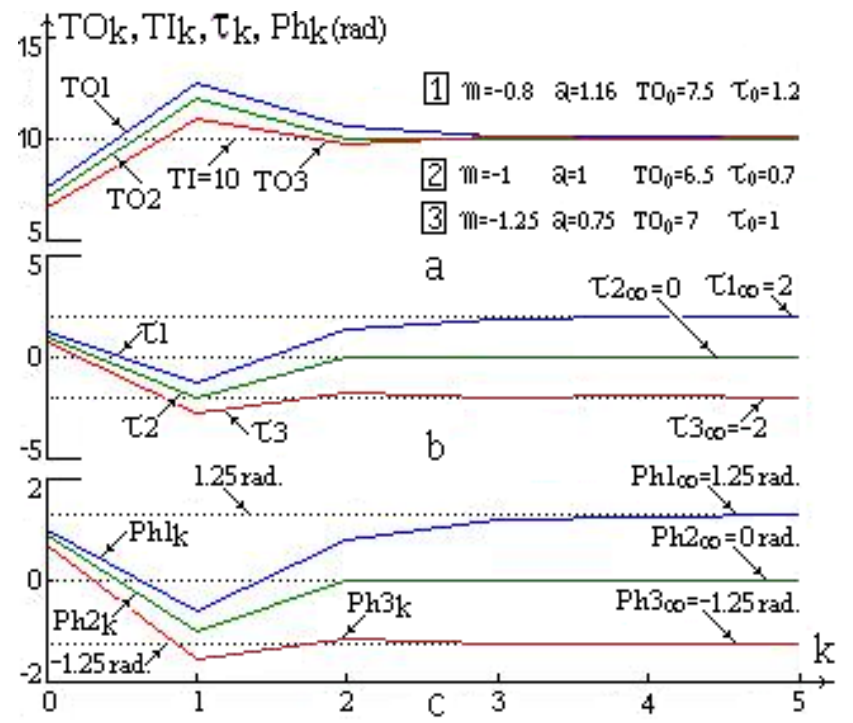

Fig. 3. Phase shifting ( $T=0$ for all of three cases) in the function of parameters " $\mathrm{m}$ " and "a": a. The output period tends to the input period, b. The final value of time difference $\tau_{\infty}=T I(1-\mathrm{a}) / \mathrm{m}, \mathrm{c}$. The final value of the phase shifting is $\mathrm{Ph}_{\infty}=2 \pi \cdot \tau_{\infty} / T O_{\infty}[\mathrm{rad}]=2 \pi \cdot(1-\mathrm{a}) / \mathrm{m}[\mathrm{rad}]$.

This agreement confirms the correctness of the simulation results and the correctness of the mathematical approach. We can now reach the general conclusion about the influence of parameter "a" to the phase shifting. If $a>1$, the phase shifting is positive. If however $a<1$, the phase shifting is negative. Since the parameter "a" does not affect the stability of the system, both of them, the positive and the negative shifting can be unlimited. The only limitation is the capacity of the circuits which are built in the shifter. The general expression for the phase shifting, for both, the time and the phase shifter, can be 
expressed as $\mathrm{Ph}_{\infty}=2 \pi \cdot \tau_{\infty} / T O_{\infty}[\mathrm{rad}]$. Changing $\tau_{\infty}=T I(1-\mathrm{a}) / \mathrm{m}$ for the phase shifter, and taking in account that for the stable shifter $T I=T O_{\infty}$, the expression turns into $\mathrm{Ph}_{\infty}=2 \pi \cdot(1-\mathrm{a}) / \mathrm{m}$ [rad]. The transition state, as well the stable state, for all of three cases of the phase shifting are presented in Figure 3c. According to the previous expression, $P h 1_{\infty}=2 \pi \cdot(1-1.16) /(-$ $0.8)=1.25[\mathrm{rad}], \quad \mathrm{Ph} 2_{\infty}=2 \pi \cdot(1-1) /(-1)=0$ [rad $]$ and $\mathrm{Ph} 3_{\infty}=2 \pi \cdot(1-$ $0.75) /(-1.25)=-1.25$ [rad]. All of calculated results agree with the simulated ones, shown in Figure 3c.

\section{The shifting in case when the input is a ramp function}

Besides the described shifting for the case when the input period is a step function, the time shifter $(\mathrm{a}=1)$ can be used for the shifting of an input pulse rate, whose period is a ramp function. To illustrate this ability, let us determine the well known velocity error, providing that the input period is the ramp function $T I(\mathrm{k})=T I_{\mathrm{V}}(\mathrm{k})=\mathrm{p} \cdot \mathrm{k}$, where " $\mathrm{p}$ " is a time constant. The definition of velocity error is $\mathrm{K}_{\mathrm{V}}=\lim \left[T O_{\mathrm{V}}(\mathrm{k})-T I_{\mathrm{V}}(\mathrm{k})\right]$ for $\mathrm{k} \rightarrow \infty$. One more convenient expression for velocity error is $\mathrm{K}_{\mathrm{V}}$ $=\lim T I_{\mathrm{V}}(\mathrm{k})\left[\mathrm{H}_{\mathrm{TO}}(\mathrm{k})-1\right]$ for $\mathrm{k} \rightarrow \infty$, where $\mathrm{H}_{\mathrm{TO}}(\mathrm{k})$ is the transfer function given by (7). Taking $\mathrm{a}=1$ and using the previous expression and $\mathrm{Z}$ transform of velocity function $T I(\mathrm{z})=T I_{\mathrm{V}}(\mathrm{z})=$ $Z(p \cdot k)=p z /(z-1)^{2}$, it can be found out that $K_{V}=0$. This means that the output of the shifter is capable to track the ramp input, without an error.

In order to consider the shifting abilities of the circuit, let us now determine the behaviour of $\tau_{V}(k)$ for the velocity (ramp) input, if $k \rightarrow \infty$. Using the final value theorem, $\tau_{V \infty}=\lim \tau_{V}(k)_{\mathrm{k} \rightarrow \infty}$ is calculated using $\tau_{\mathrm{V}}(\mathrm{z})$ and shown in (14). The expression $\tau_{V}(z)$ is get out by the substitution of $T I(z)=T I_{V}(z)$ in (6) and taking $\mathrm{a}=1$. According to (14), $\tau_{V \infty}$ is the time constant, which does not depend on the initial conditions. Besides the parameters "m" and " $T$ ", $\tau_{V \infty}$ depends on the time constant "p",

$$
\tau_{V \infty}=\lim \left[(z-1) \tau_{V}(z)\right]_{z \rightarrow 1}=(p-T) / m
$$

which is the slope of the ramp input function. It means the time shifting of the output pulses can be controlled by the control word " $T$ ". If $T>\mathrm{p}, \tau_{V \infty}>0$ and the output pulse leads in time, just like in Figure 1. If however $\mathrm{T}<\mathrm{p}, \tau_{V_{\infty}}$ is negative and the output pulse is delayed in time, in comparison with the corresponding input pulse. It comes out that if $T=\mathrm{p}, \tau_{V_{\infty}}=0$, i.e. the shifter functions as classic PLL, reducing the phase difference to zero, even for the ramp input function. To check the correctness of the obtained results, the simulations of $T O(\mathrm{k})$ and $\tau(\mathrm{k})$ for the velocity input $T I_{\mathrm{k}}=(10+4 \cdot \mathrm{k})$ t.u. are shown in Figure 4 . Three cases for different parameters " $\mathrm{m}$ " and " $T$ " are presented. All initial conditions and final values are shown in Figure 4. For all of cases in Figure 4a, the output periods track the input periods without an error. That means $\mathrm{K}_{\mathrm{V}}=0$, which agrees with the previous theoretical conclusion. Note that the shifter takes only two steps to reach the stable state for $m=-1$, regardless of the fact that the input is the ramp function. For all cases in Figure $4 \mathrm{~b}$, time differences tends to the time constant $"(\mathrm{p}-T) / \mathrm{m}$ ", given by (14), proving the correctness of the mathematical analyses. For case Nr. $2, \mathrm{~T}=\mathrm{p}=4$ t.u. and according to (14), $\tau_{\mathrm{V} 2 \infty}=0$. That means the shifter predicts and generates the output period which is completely synchronized with the input pulses, even for the case when the input is a ramp function. For case Nr. 1,
$\tau_{\mathrm{V} 1 \infty}=(\mathrm{p}-T) / \mathrm{m}=(4-7.75) /(-0.75)=5$ t.u. For case Nr. $3, \tau_{\mathrm{V} 3 \infty}=(\mathrm{p}-$ $T) / \mathrm{m}=[4-(-2)] /(-1.25)=-4.8$ t.u. The calculated results agree with the simulated ones, shown in Figure 4b. Cases Nr. 1 and Nr. 3 prove that the shifter is able to generate both positive and negative shifting of the pulses of a ramp input, depending on the parameter " $T$ ". For case $\mathrm{Nr} .1 T>\mathrm{p}\left(\tau_{\mathrm{V} \infty}>0\right)$, the output pulses lead for $\tau_{\mathrm{V}^{\circ}+}$ in comparison with the input pulses. For the case Nr. 3, $T<\mathrm{p}\left(\tau_{\mathrm{V}_{\infty}}<0\right)$, the output pulses are delayed for $\tau_{\mathrm{V} \infty}$ in comparison with the input pulses.

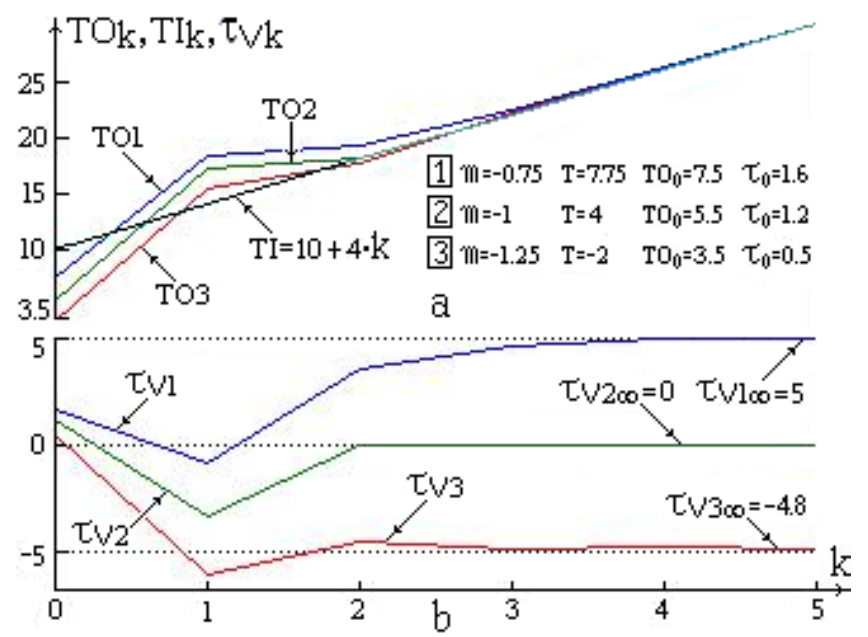

Fig. 4. The time shifting of the ramp input function $(\mathrm{a}=1)$ : $a$. The output periods, for all of three cases, track the input periods without an error, $\mathrm{Kv}=0$, b. The time shiftings tend to the time differences $\tau_{V_{\infty}}$, given by eq. (14).

\section{REALIZATION OF THE SHIFTER}

According to the results obtained, the optimum value of " $\mathrm{m}$ " for the shifting applications, is $\mathrm{m}=-1$. Firstly, the shifter is the fastest for $m=-1$ and secondly the realization of the shifter is much simpler if $m=-1$. For some other applications, where it is necessary to adapt the locking speed, different values of " $\mathrm{m} "$ can be required. Taking $m=-1$, and changing $a=f_{a} / f_{c}$, where $f_{a}$ and $f_{c}$ are the clock frequencies, (2) turns into (15). According to (15), $T_{k}$ and $\tau_{\mathrm{k}+1}$ are measured by the clock period $\mathrm{t}_{\mathrm{c}}=1 / \mathrm{f}_{\mathrm{c}}, T I_{\mathrm{k}}$ is measured by the clock period $\mathrm{t}_{\mathrm{a}}=1 / \mathrm{f}_{\mathrm{a}}$ and $T O_{\mathrm{k}+1}$ is generated by the clock period $t_{c}=1 / f_{c}$. The principal scheme of the realization, corresponding to (15) is shown in Figure 5.

$$
f_{c} \cdot T O_{k+1}=f_{a} \cdot T I_{k}+f_{c} \cdot T_{k}-f_{c} \cdot \tau_{k+1}
$$

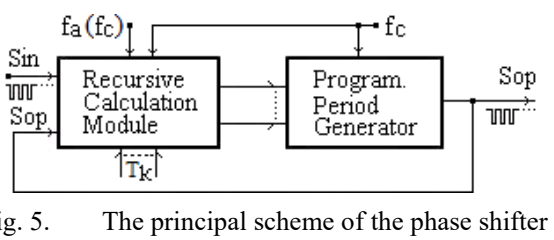

The shifter consists of a Recursive Calculation Module (RCM) and a Programmable Period Generator (PPG). RCM calculates $\mathrm{TO}_{\mathrm{k}+1}$ and PPG generates the output period at the next step. PPG was described in detail in [1-9]. The realization of RCM depends on the type of algorithm, but the realization of 
RCM according to (15), can be easily performed using the technique described in [1-9]. According to the previous conclusions, if $\mathrm{f}_{\mathrm{a}}=\mathrm{f}_{\mathrm{c}}(\mathrm{a}=1)$, the circuit functions as time shifter. If however $\mathrm{f}_{\mathrm{a}}$ is not equal to $\mathrm{f}_{\mathrm{c}}$ and $T_{\mathrm{k}}=0$, the circuit functions as the phase shifter. One eight bits shifter was realized by standard integrated circuits for $\mathrm{a}=1\left(\mathrm{f}_{\mathrm{a}}=\mathrm{f}_{\mathrm{c}}\right), T=0$ and $\mathrm{m}=-1$. The input period, shown in Figure 6, is the step function $T I=1[\mathrm{~ms}]$, corresponding to the frequency of $1[\mathrm{kHz}]$. The clock frequency $\mathrm{f}_{\mathrm{c}}=6[\mathrm{kHz}]$, so that the ratio $T I / \mathrm{t}_{\mathrm{c}}=6$. The oscilloscope picture of the voltage waveforms, shown in Figure 6, was taken when the shifter was in the stable state. In accordance with the chosen parameters, the shifter functions as a classic PLL, striving to reduce the time difference to zero. However, that is not possible because the ratio $T I / \mathrm{tc}$ is very small. The ratio $T I / \mathrm{tc}$ is chosen intentionally small to enable the visibility of $\tau_{\infty++}$ and $\tau_{\infty}$ and to provide the evidence of the whole physical process. In accordance with the chosen parameters, the time difference $\tau_{\infty}$ is changing the sign at every step. If $T I / t_{\mathrm{c}}$ increases, the widths of $\tau_{\infty \circ+}$ and $\tau_{\infty \text { - }}$ would decrease and tend to zero. It can also be seen in Figure 6 that whenever two pulses of Sop occur during a period $T I$, it means that $T I>T O$, and the coming " $\tau_{\infty}$ " is negative. If two pulses of Sin comes during a period $T O$, it means that $T O>T I$, and the coming " $\tau_{\infty}$ " is positive. The previous explanations, at the same time, contribute to the understanding of the principles of the shifter functioning.

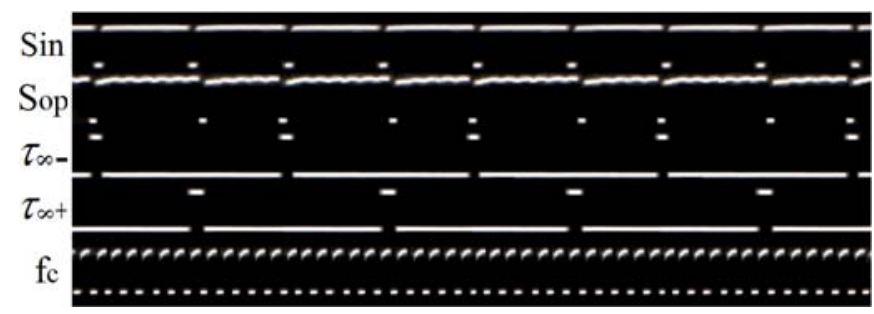

Fig. 6. The shifter is in the stable state. It functions as PLL $(a=1, m=1$, $T=0$ ). The ratio $T I / \mathrm{t}_{\mathrm{c}}$ is only about six and the time differences $\tau_{\infty+}$ and $\tau_{\infty \text { - }}$ are visible. If $T I / \mathrm{t}_{\mathrm{c}}$ increases, the time differences tend to zero.

\section{CONCLUSION}

The description and illustration of the realized shifter represent one additional contribution to the shifters described in $[1,2]$. The presented theoretical approach in whole, also represents the contribution to the field of TRP. It was demonstrated that besides the processing of the input and output periods and the time differences between them, an additional control function can take part in the calculation of the output period. In dependence on the type of the control function, the shifter takes new properties. For instance, it was shown that if the additional control function is a step function, the shifter is able to provide the proper shifting even in case the input period is a ramp function. This example indicates that, using this approach, it is possible to develop new additional applications of the circuits and systems based on TRP.

In comparison with the shifters described in $[1,2]$, this shifter provide important advantages consisting of the better functionality and the wider applicability. Firstly, unlike the mentioned shifters, this time shifter provides both the time shifting and the phase shifting. Secondly, the time shifting does not depend on the input period, so that the desired value of either positive or negative shifting is directly proportional to the outside control word. This property significantly facilitates its practical realization and its practical usage. Thirdly, this shifter can generate either a positive or negative shifting of the input pulse signal, even in case the input period is a ramp function. This feature provides a much wider field of application.

It was shown that if $T=0$, the shifter can function exactly as classic PLL, which has already found a number of applications. The analysis and simulations showed that this phase shifter is extremely fast if $m=-1$. In this case, the shifter takes only two steps to reach the stable state. Changing $\mathrm{m}$ in range of -2 to 0 , it is possible to adapt a transient time of the shifter to the required application. The precision of the phase shifting can be extremely high. It is limited however by the speed of the used integrated circuits, since it depends on relation $T I / \mathrm{t}_{\mathrm{c}}$. A higher $T I / \mathrm{t}_{\mathrm{c}}$ relation provides a higher shifting resolution. Generally, the shifting band can be as large as necessary. However, the capacity of the built-in up-down counters must be large enough to satisfy the requirements for the shifting band and the shifter resolution. The identity of the analytical and simulation results, in every step, proves the correctness of the entire theoretical approach, as well as the validity of the obtained results.

\section{REFERENCES}

[1] D. Perisic, M. Perisic, S. Rankov, "Phase shifter based on a Recursive Phase Locked Loop of the second order", Rev. Roum. Sci. Techn.Electrotechn. et Energ., Vol. 59, No. 4, pp. 391-400, 2014

[2] D. Perisic, A. Zoric, D. Babic, D. Perisic, "Recursive PLL of the First Order”, Przegląd Elektrotechniczny, Vol. 89, No.7, pp. 50-53, 2013

[3] D. Perisic, A. Zoric, S. Obradovic, D. Perisic, "FLL as digital period synthesizer based on Binary Rate Multiplier control", Przegląd Elektrotechniczny, Vol. 89, No. 1a, pp. 145-148, 2013

[4] D. Perisic, M. Bojovic, "Multipurpose Time Recursive PLL ", Rev. Roum. Sci. Techn.-Electrotechn. et Energ., Vol. 61, No. 3, pp. 283-288, 2016

[5] D. Perisic, A. Zoric, M. Perisic, D. Mitic, "Analysis and Application of FLL based on the Processing of the Input and Output Periods", Automatika, Vol. 57, pp. 230-238, 2016

[6] D. Perisic, M. Perisic, D. Mitic, M. Vasic "Time Recursive Frequency Locked Loop for the tracking applications", Rev. Roum. Sci. Techn.Electrotechn. et Energ., Vol. 60, No. 2, pp. 195-203, 2015

[7] D. Perisic, A. Zoric, M. Perisic, V. Arsenovic, L. Lazic "Recursive PLL based on the Measurement and Processing of Time", Elektronika ir Elektrotechnika, Vol. 20, No. 5, pp. 33-36, 2014

[8] D. Perisic, A. Zoric, D. Babic, D. Perisic, "Decoding and Prediction of Energy state in Consumption control", Rev. Roum. Sci. Techn.Electrotechn. et Energ., Vol. 58, No. 3, pp. 263-272, 2013

[9] D. Perisic, A. Zoric, S. Obradovic, S. Spalevic, "Application of Frequency Locked Loop in Consumption Peak Load Control”, Przegląd Elektrotechniczny, Vol. 88, No. 1b, pp. 264-267, 2012

[10] R. N. Dean, A. K. Rane, "Digital Frequency-Locked Loop System for Capacitance Measurement", IEEE Transactions on Instrumentation and Measurement, Vol. 62, No. 4, pp. 777-784, 2013

[11] M. Abdul-Niby, M. Alameen, H.Baitie, "A Simple Phase Shifting Technique for an Injection Locked Oscillator" Engineering, Technology \& Applied Science Research, Vol. 6, No. 6, pp. 1303-1306, 2016

[12] D. Jovcic, "Phase locked loop system for FACTS", IEEE Transactions on Power Systems, Vol. 18, No. 3, pp. 2116-2124, 2003 
[13] D. Abramovich, "Phase-locked loops: a control centric tutorial", American Control Conference, Vol 1, pp. 1-15, USA, November 07, 2002

[14] A. S. N. Mokhtar, M. B. I. Raez, M. Marufuzzaman, M. A. M. Ali, "Hardware implementation of a high speed inverse park transformation using cordic and pll for foc brushless servo drive", Elektronika ir Elektrotechnika, Vol. 19, No. 3, pp. 23-26, 2013

[15] M. Pastor, J. Dudric, "Grid-tied 15-level Cascade Inverter with Predictive Current Control”, Elektronika ir Elektrotechnika, Vol 18, No. 9, pp. 19-22, 2012

[16] G. Bianchi, Phase-Locked Loop Synthesizer Simulation, Nc-Hill, Inc. New York, USA, 2005

[17] R. Vich, Z Transform Theory and Application (Mathematics and Applications), Springer, 1987

[18] D. B. Tablot, Frequency Acquisition Techniques for Phase Locked Loops, Wiley-IEEE Press, 2012

\section{ACKNOWLEDGEMENT}

This article was supported by the Ministry of Science and Technology of the Republic of Serbia within the project TR 32047.

\section{AUTHORS PROFILE}

Djurdje Perisic was born in Atenica near Cacak, Serbia in 1943. He graduated from the Faculty of Electrical Engineering in Belgrade in 1967. Through an English government scholarship, he received an M.Sc. from the Cranfield Institute of Technology, England in 1976. He received a Ph.D. at the Technical Academy in Zagreb in 1983. After that he was awarded twice the Fulbright and once the Humboldt scholarship. He was awarded the "Tesla's award", the highest
Yugoslav recognition for contributions to the field of natural sciences, from the Serbian Academy of Sciences in 1996. In 2003, as the dean of the faculty, he founded the first study programs for the Faculty of Information Technology at SPU. In 2013 he was elected a professor emeritus. His current research interests include the development of new approaches to electronic circuits and systems combining electronics, system theory and other fields. During last five years he has published fifteen papers in SCI list journals and about 40 additional papers of international importance.

Miroslav Bojovic was born in 1957. All his formal education, including the Ph.D. (1989) are from the Faculty of Electrical Engineering, University of Belgrade, Serbia. He realized the specialization at the faculty of Computer Science Department, UCLA, Los Angeles, USA, during 1988 and 1989. As a Head of the Computer Science Department at the Faculty, he is currently engaged as a professor at the Faculty of Electrical Engineering in Belgrade in the field of Database menagement System and Software Engineering and as a project leader of many projects in Software engineering. One of his projects "MobilePDR" was awarded as the best software product in USA in the field of medical information systems in 2004. As a software product, it has been referenced in more than 100,000 different publications, all over the world. 